\title{
The Role of the Wnt Signaling Pathway in Upper Jaw Development of Chick Embryo
}

\author{
Tadahiro Shimomura ${ }^{1}$, Masayoshi Kawakami ${ }^{1}$, Kouko Tatsumi ${ }^{2}$, Tatsuhide Tanaka ${ }^{2}$, \\ Shoko Morita-Takemura ${ }^{2}$, Tadaaki Kirita ${ }^{1}$ and Akio Wanaka ${ }^{2}$ \\ ${ }^{1}$ Department of Oral and Maxillofacial Surgery, Nara Medical University, 840 Shijo-cho, Kashihara, Nara 634-8521, Japan \\ and ${ }^{2}$ Department of Anatomy and Neurosciences, Nara Medical University, 840 Shijo-cho, Kashihara, Nara 634-8521, Japan
}

Received October 22, 2018; accepted December 29, 2018; published online February 23, 2019

\begin{abstract}
Cleft lip with or without cleft palate (CLP) usually results from a failure of the medial nasal prominences to fuse with the lateral and maxillary prominences. This failure inhibits facial morphogenesis regulated by several major morphogenetic signaling pathways. We hypothesized that CLP results from the failure of the Wnt signaling pathway. To examine whether Wnt signaling can influences upper jaw development, we applied beads soaked with Dickkopf-1 (Dkk-1), Alsterpaullone (AL) or Wnt3a to the right side of the maxillary prominence of the chick embryo. The embryo showed a defect of the maxilla on the treated side, and skeletal staining revealed hypoplasia of the premaxilla and palatine bone as a result of Dkk-1-soaked bead implantation. 5-bromo-2'-deoxyuridine (BrdU)-positive cell numbers in the treated maxillary prominence were significantly lower at both 24 and $48 \mathrm{hr}$ after implantation. Down-regulation of the expression of Bmp4, Tbx22, Sox9, and Barx1 was confirmed in the maxillary prominence treated with Dkk-1, which indicated that the deformity of the maxillary bone was controlled by gene targets of the Wnt signaling pathway. Expression of $\mathrm{N}$-cadherin was seen immunohistochemically in the maxillary prominences of embryos at $6 \mathrm{hr}$ and increased at $24 \mathrm{hr}$ after AL treatment. Wnt signaling enhanced by AL or Wnt3a up-regulated the expression levels of Msx1, Bmp4, Tbx22, Sox9, and Barx1. Our data suggest that the Wnt signaling pathway regulates maxillary morphogenesis and growth through Bmp4, Tbx22, Sox9, and Barx1. Wht signaling might regulate $\mathrm{N}$-cadherin expression via Msx1, resulting in cell aggregation for osteochondrogenesis.
\end{abstract}

Key words: Wnt signaling, Dickkopf-1, N-cadherin, cleft lip, chick embryo

\section{Introduction}

Cleft lip with or without cleft palate (CLP) is one of the most common birth defects in humans [3,25]. CLP usually results from a failure of the medial nasal prominences to fuse with the lateral and maxillary prominences, which inhibits growth and development. Facial development is regulated by several major morphogenetic signaling pathways, such as Wnt, FGF, and BMP signaling [11, 28, 34, 38].

The Wnt signaling pathway is one of the prominent

Correspondence to: Masayoshi Kawakami, DDS, Ph.D., Department of Oral and Maxillofacial Surgery, Nara Medical University, 840 Shijo-cho, Kashihara, Nara 634-8521, Japan. E-mail: mkawaka@naramed-u.ac.jp intercellular signaling pathways and plays fundamental roles in cellular proliferation, regeneration, and differentiation. In mice, several Wnt ligands, including Wnt 3 and $W n t 9 b$, are expressed in the ectoderm on the early facial prominences [20]. Variation in Wnt signaling is tightly associated with non-syndromic CLP [6]. The signaling activity is initiated by Wnt ligands binding to a dual receptor complex, LRP5/6, resulting in the stabilization and nuclear translocation of $\beta$-catenin, which further binds with TCF/LEF to regulate the transcription of downstream target genes. WNT signaling activity is negatively regulated by secreted proteins such as Dickkopf-1 (Dkk-1). Dkk-1 dampens signaling activity by disengaging LRP from the receptor complex, thereby blocking the WNT ligand- 
receptor interaction [9]. In the absence of Wnt signaling, free $\beta$-catenin is ubiquitinated and degraded by the proteasome. Mice deficient for LRP6 receptor exhibited cleft lip with cleft palate, resembling the common birth defect CLP in humans [31]. Ablation of Wnt secretion in the ectoderm leads to a failure of the frontonasal and maxillary prominences to form [38]. The frontonasal and upper jaw prominences do not form when $\beta$-catenin is conditionally ablated in the facial ectoderm $[18,28,37]$. However, the function of Wnt signaling in craniofacial development is poorly understood.

Interaction between Wnt signaling and other signaling pathways may be important for craniofacial development. Bone morphogenetic protein (BMP) signaling is known to be important for growth of facial prominences by controlling the expression of Sox9 and Runx2 in the maxillofacial region $[5,23,36]$. While BMP signaling mainly affects proliferation, fibroblast growth factor (FGF) signaling in the cranial frontonasal mass is required to maintain cell proliferation and cell survival [33]. BMP and FGF in the frontonasal mass regulate $T b \times 22$ expression, which can contribute to CLP [12]. Wnt $9 b$ gene expression positively regulates FGF gene expression in the ectoderm of the maxillary prominence, and is required for the proliferation of mesenchymal cells of the maxillary prominence in mice [14].

Wnt signaling plays crucial roles in embryonic development, but mechanisms of Wnt signaling function in the facial prominence are not well characterized. It is likely that decreased signaling function can explain all of the mechanisms underlying CLP. We hypothesize that CLP results from a failure in development and growth of the facial prominence due to reduced Wnt signaling. In this study, we used chick embryos and examined whether the Wnt signaling pathway is required for maxillofacial development. To test the hypothesis, we used Dkk-1 as an antagonist and investigated morphological changes and gene expression in the developing upper jaw and lip.

\section{Materials and Methods}

\section{Embryos and bead implantation}

Fertilized white leghorn eggs were obtained from Takeuchi Farm (Nara, Japan) and incubated at $38^{\circ} \mathrm{C}$ until the embryos reached the appropriate stage [10]. Affi-Gel Blue beads (Bio-Rad, Hercules, CA, USA) were soaked in $0.1 \mathrm{mg} / \mathrm{ml} \mathrm{Dkk-1} \mathrm{(Abcam,} \mathrm{Cambridge,} \mathrm{UK),} 0.5 \mathrm{mg} / \mathrm{ml}$ Noggin (R\&D Systems, Minneapolis, MN, USA) or 1.0 $\mathrm{mg} / \mathrm{ml}$ Wnt3a (R\&D Systems, Minneapolis, MN, USA) with $2 \%$ bovine serum albumin (BSA). AG1-X2 beads (Bio-Rad, Hercules, CA, USA) was in $10 \mathrm{mg} / \mathrm{ml}$ Alsterpaullone (AL) (Sigma-Aldrich, St Louis, MO, USA) in DMSO for $1 \mathrm{hr}$ with a drop of $0.01 \%$ Fast Green added for bead visualization. Sham operations were performed using beads soaked in $2 \%$ BSA. In all cases, the beads were inserted on right side of the maxillary prominence by making small incisions at stage 22 (before the fusion of the frontonasal prominence and maxillary prominence) under a microscope (Leica MZ7.5, Wetzlar, Germany). After bead implantation, the embryos were reincubated for additional periods ranging from $6 \mathrm{hr}$ to 12 days (stage 38). Animal procedures were approved by the Nara Medical University Animal Care and Use Committee.

\section{Bromodeoxyuridine labeling and immunofluoresence staining}

After incubation for 6,24 , or $48 \mathrm{hr}$, the beadimplanted embryos were injected with $10 \mu \mathrm{l}$ of 5-bromo-2'deoxyuridine (BrdU) (Sigma-Aldrich, St Louis, MO, USA) $2 \mathrm{hr}$ before euthanization. They were then fixed in $4 \%$ paraformaldehyde and processed in paraffin. Paraffin sections were pretreated with $2 \mathrm{~N} \mathrm{HCl}$ for 5 min after antigen activation in $0.1 \mathrm{M}$ sodium citrate buffer at $95^{\circ} \mathrm{C}$ for $10 \mathrm{~min}$. The slides were incubated with rat anti-BrdU monoclonal antibody $(1: 100)$.

Another set of embryos were treated with Dkk-1- or AL-soaked beads at stage 20 and collected 24 or $48 \mathrm{hr}$ after implantation. Antigen retrieval for anti-N-cadherin antibody was performed by incubating the beads in $0.1 \mathrm{M}$ sodium citrate buffer for $10 \mathrm{~min}$ at $95^{\circ} \mathrm{C}$. Rabbit polyclonal antibody to N-cadherin (1:200; Abcam, Cambridge, UK) was applied overnight at $4^{\circ} \mathrm{C}$. For immunohistochemical staining, biotinylated anti-rat IgG $(\mathrm{H}+\mathrm{L})(\mathrm{BA}-9400$, Vector Laboratories, Burlingame, CA, USA) and were applied for $1 \mathrm{hr}$ at room temperature. Immunofluorescence sections were incubated with Alexa Flour 488-conjugated goat antirabbit $\operatorname{IgG}(\mathrm{H}+\mathrm{L})$ (Invitrogen, $\mathrm{CA})$. The sections were coverslipped in VECTASHIELD Hard Set Mounting Medium with DAPI (Vector Laboratories, Burlingame, CA, USA). Images were acquired using a confocal fluorescence microscope (C2/Ni-E, Nikon, Tokyo, Japan).

\section{Real-time RT-PCR}

After incubation for $6 \mathrm{hr}$, the maxillary prominence of the bead-treated side was dissected and stored in RNA later (Ambion, Austin, TX, USA) ( $\mathrm{N}=6-8$ for each experiment). Total RNA was isolated with Sepasol-RNA I Super $\mathrm{G}$ (Nacalai Tesque, Kyoto, Japan) from the dissected processes. We synthesized cDNA using a QuantiTect Reverse Transcription Kit (Qiagen, Mainz, Germany), and chicken cDNA was prepared as previously described [18]. The polymerase chain reaction (PCR) primers used were as previously described $[12,18,20,29,32,38]$. Semiquantitative RT-PCR was performed according to the manufacturer's protocol. At least six processes were included in one sample, and three samples were examined in each quantitative analysis for statistical significance.

\section{Skeletal staining}

To study morphological changes of bone and cartilage, embryos were fixed at stage 38 (after 12 days' incubation) and kept in $100 \%$ ethanol for 4 days. The embryos were 
then infiltrated with acetone for 4 days, stained with Alcian blue and Alizarin red for up to 10 days, and cleared with $\mathrm{KOH}$ as previously described [18].

\section{Statistical analysis}

The delta-delta CT method and ANOVA using Microsoft Excel were carried out for statistical comparisons, and a value of $P<0.05$ was considered significant.

\section{Results}

\section{External and skeletal effects of Dkk-1-soaked bead implantation}

Dkk-1 is an antagonist of the canonical Wnt signaling pathway by reducting in $\beta$-catenin $[7,18,22]$. To determine whether direct antagonism of Wnt signaling can affect upper jaw development, we applied Dkk-1-soaked beads to the maxillary prominence of stage 22 chick embryos, which were then incubated until they reached stage 38 (Fig. 1AD). The embryo heads showed a defect of the maxilla on the treated side (Fig. 1B, D). Control embryos treated with BSA-soaked beads displayed no morphological changes or effects of surgery on chick development. Skeletal staining with Alcian blue and Alizarin red showed hypoplasia of the premaxilla and palatine bone following Dkk-1-soaked bead implantation (Fig. 1F, H). Control embryos treated displayed no hypoplasia of bone (Fig. 1E, G). Thus, these results suggested that blocking Wnt signaling could interfere with upper jaw development and lead to a failure to form the premaxilla and palatine bone.

\section{Wnt signaling contributes to maintaining cell proliferation in the maxillary prominences}

In a previous study, we observed cell death at $6 \mathrm{hr}$ after Dkk-1 treatment, although it decreased at $24 \mathrm{hr}$ and no apoptotic cells were seen at $48 \mathrm{hr}$ after treatment [18]. We compared cell proliferation in embryos treated with Dkk-1 and in that treated with BSA (control) at stage 22 (Fig. 2A). Fewer BrdU-positive cells were seen in the treated embryos than in the controls and cell proliferation was reduced around the Dkk-1-treated (Fig. 2B). At $6 \mathrm{hr}$ after Dkk-1 bead implantation the embryos displayed no change in proliferation around the bead compared with BSA-treated embryos. After 24 and 48 hr, however, fewer BrdU-positive cells were seen in the treated embryos than in the controls and the number of BrdU positive cells was significantly decreased rather than the control $(P<0.05)$ (Fig. 2C). These results therefore indicate that inhibition of Wnt signaling activity decreases cell proliferation in the maxillary mesenchyme, which may lead to a reduction of maxillary growth.

\section{Dkk-1 inhibits gene expression in the maxillary prominences}

We have already found that blocking the Wnt signaling pathway by Dkk-1 down-regulated Msxl, and Msx2 expression [18]. Moreover, as shown in Fig. 3, Bmp4,

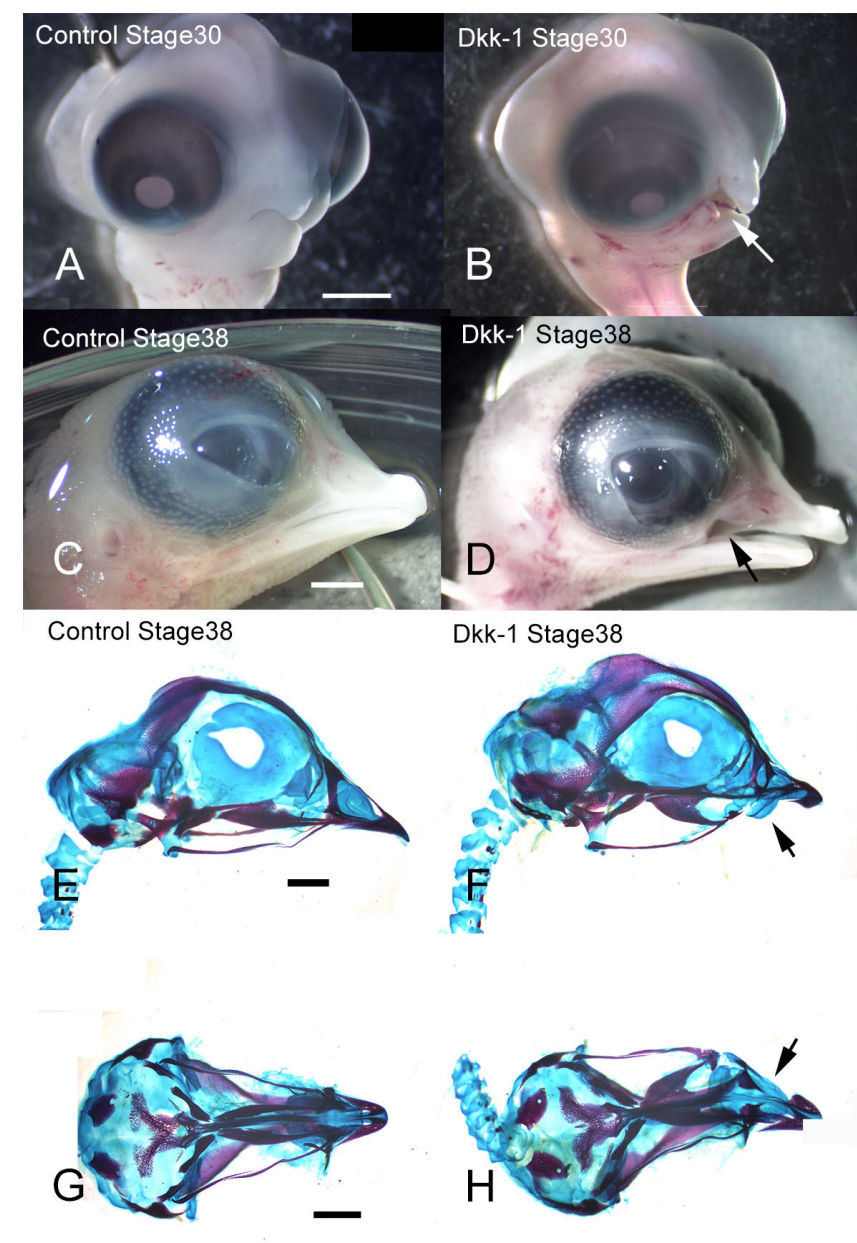

Fig. 1. Skeletal and external changes of chick face after Dkk-1-soaked bead implantation. Beads were implanted into the right side of the maxillary prominences at stage 22 and the embryos were fixed at stage 30 or stage 38 (A, B, C, D). Control embryos $(\mathbf{A}, \mathbf{C})$ were treated with $2 \%$ BSA-soaked beads. Dkk-1-soaked beads caused a defect of the maxilla at stage 30 (B, white arrow) and stage 38 (D, black arrow). Lateral $(\mathbf{E}, \mathbf{F})$ or axial $(\mathbf{G}, \mathbf{H})$ views of heads visualized with the skeletal stains Alcian blue and Alizarin red are shown at stage 38 . Hypoplasia of the premaxilla and palatine bone occurred (black arrows in $(\mathbf{F})$ and $(\mathbf{H})$, respectively). Bars $=5 \mathrm{~mm}$.

Sox9, Tbx22, and Barxl gene expression is significantly decreased by Dkk-1-soaked bead implantation in the maxillary prominences of the embryo. On the other hand, Wnt $3 a$, Bmp7, Smad1, Fgf8b, Fgf17, and Tbxl mRNA levels were not significantly different from BSA-treated controls (data not shown). To identify effects on the facial mesenchyme of blocking Wnt signals, we treated embryos with AL to see whether the blocking could be rescued. AL is a potent inhibitor of the Wnt signaling pathway component glycogen synthase kinase-3- $\beta$ (GSK3 $\beta$ ), which is implicated in the degradation of $\beta$-catenin and it is activated by an increase in intracellular $\beta$-catenin as a Wnt signal effector [4]. These AL treatment up-regulated the depression of Bmp4, Sox9, Tbx22 and Barxl after Dkk-1 treatment, 
A

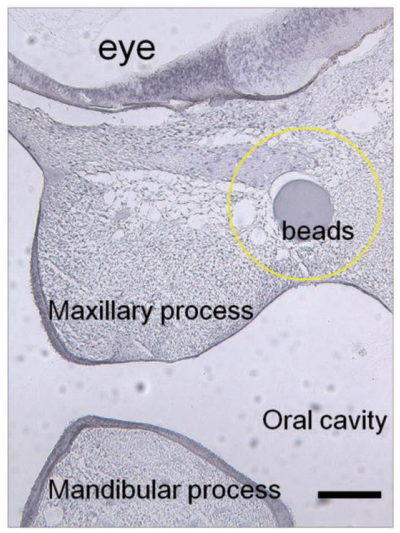

B

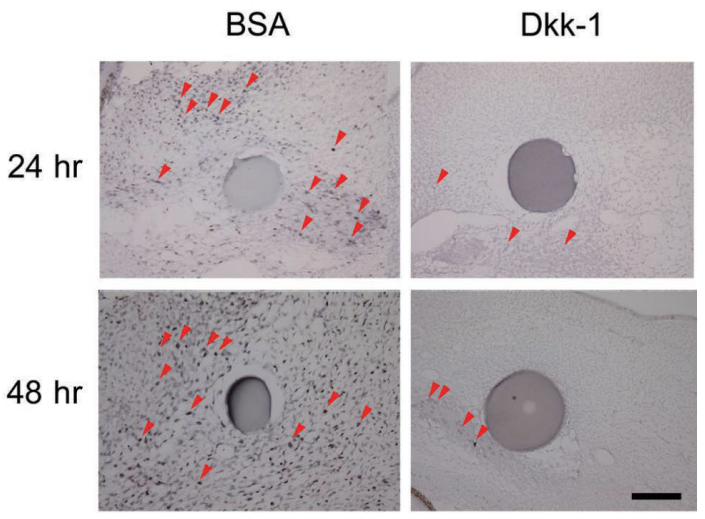

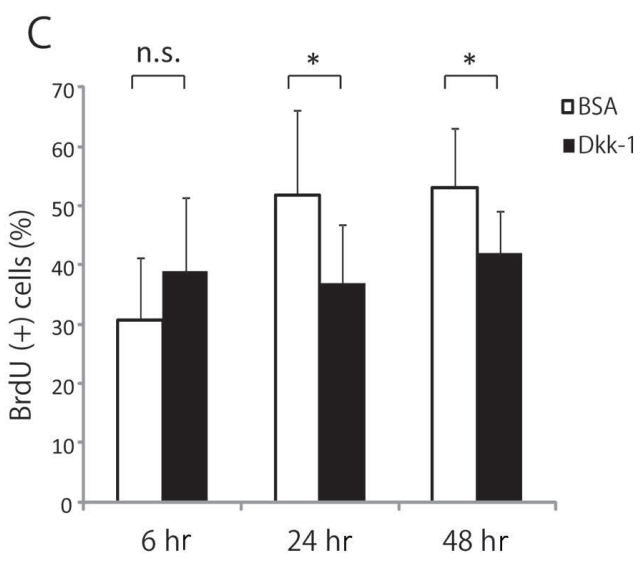

Fig. 2. BrdU labeling and immunohistochemical staining to evaluate cell proliferation. (A) The area in which BrdU-positive cells were counted around an implanted bead (within a radius of $100 \mu \mathrm{m}$ ) is indicated by a yellow circular line. (B) BrdU-labeled cells (red arrowheads) were more abundant around BSA-treated beads $(n=5)$ than around Dkk-1-treated beads $(n=5)$. Bars $=100 \mu \mathrm{m}$. $(\mathbf{C})$ The statistical results of BrdU-positive cells in the maxillary prominences treated with BSA and Dkk-1 after $6 \mathrm{hr}, 24 \mathrm{hr}$, and $48 \mathrm{hr}$. Values are the mean \pm S.D. in separate experiments using 9 embryos. *: $P<0.05$.

which did not reached any significant difference levels from control (Fig. 3). These results confirmed that the Wnt signal could control the expression of Bmp4, Sox9, $T b \times 22$, and Barxl.

\section{Effect of inhibiting BMP in the maxillary prominences of the embryo}

As blocking of Wnt signaling reduced Bmp 4 expression, we assessed the expression of several genes in the maxillary mesenchyme where BMP signaling was locally blocked. Noggin is a potent BMP antagonist, and blocks the osteo-inductive potential of BMPs by forming complexes that inhibit the ability of BMPs to interact with their receptors $[5,36]$. Noggin-treated embryos had significantly reduced expression of Sox9. However, Noggin treatment did not significantly influence $B m p 7$ and $F g f 8 b$ expression. On the other hand, Bmp4 and Wnt $3 a$ mRNA expression was upregulated by Noggin-soaked bead implantation in the maxillary prominences of the embryo (Fig. 4).

\section{Enhanced Wnt signaling leads to $\mathbf{N}$-cadherin expression and up-regulated gene expressions in the maxillary prominence}

Our study confirmed that inhibition of Wnt signaling down-regulated the gene expression of $B m p 4, \operatorname{Sox} 9, T b \times 22$ and Barxl. Next, we examined a gain-of-function of Wnt signaling by $\mathrm{AL}$ in the maxillary prominence. AL application increased $M s x 1$ expression after $6 \mathrm{hr}$, but not Msx2, Tbx22, Sox9 (data not shown). Immunohistochemical analysis showed $\mathrm{N}$-cadherin was expressed in the extracellular space and aggregated around the implanted beads in the maxillary prominences. The labeling of $\mathrm{N}$-cadherin increased at $24 \mathrm{hr}$ after AL treatment (Fig. 5). Combined the immunochemical results, it was implied that enhanced Wnt signaling induced $\mathrm{N}$-cadherin expression in the maxil- 


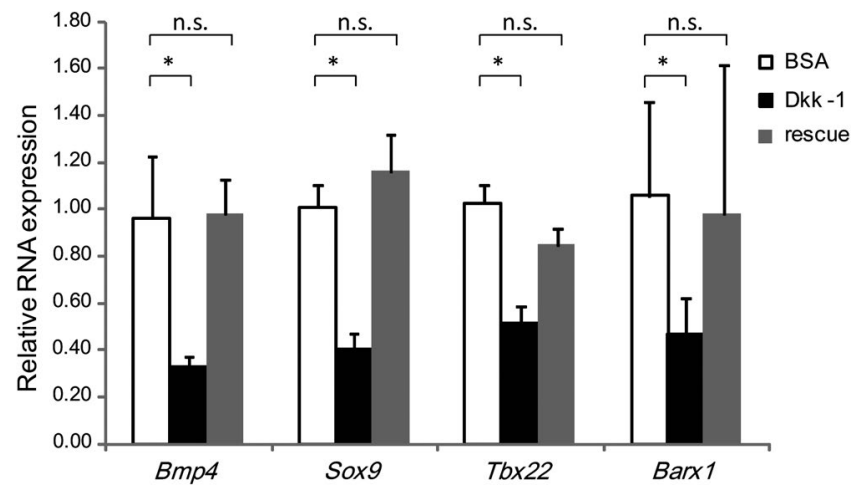

Fig. 3. Real-time RT-PCR analysis in chick maxillary mesenchyme treated with Dkk-1 and rescued with AL. The chart shows real-time RTPCR results for different genes from maxillary mesenchyme treated with Dkk-1 and AL. Each bar shows BSA treated sample on the left, Dkk-1 treated sample on the middle, and rescue situation on the right. Bmp4, Sox9, Tbx22, and Barxl gene expression significantly decreased following Dkk-1-soaked bead implantation in the maxillary process. Bmp4, Sox9, Tbx22, and Barxl mRNA levels were rescued by combined treatment with Dkk-1 and AL and showed no significant difference from BSA treatment. Values are the mean \pm S.E. in separate experiments using 9 embryos. *: $P<0.05$, significantly different from the corresponding value for BSA treated group.

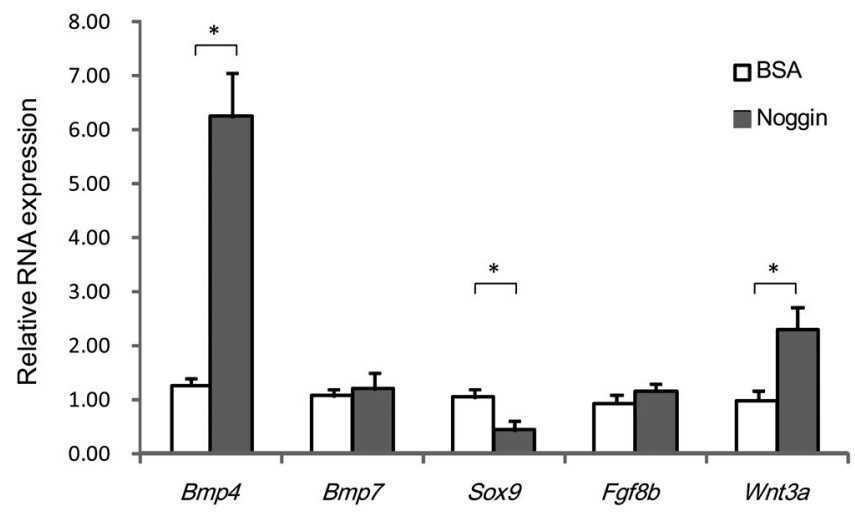

Fig. 4. Real-time RT-PCR analysis after application of Noggin in chick maxillary mesenchyme. The chart shows real-time RT-PCR results for different genes from maxillary mesenchyme treated with BSA and Noggin. Each bar shows BSA treated sample on the left and experimental situation on the right. Bmp 4 and $W n t 3 a$ gene expression significantly increased after Noggin-soaked bead implantation in the maxillary prominences, whereas Sox 9 gene expression decreased. Values are the mean \pm S.E. in separate experiments using 9 embryos. *: $P<0.05$, significantly different from the corresponding value for BSA treated group.

lary prominence via $M s x 1$.

Furthermore, we applied Wnt3a to the maxillary prominence as the Wnt-protein ligand. We identified that the expressions of Msx1, Bmp4, Tbx22, Sox9, and Barxl were up-regulated by at least 3.4-, 4.3-, 5.9-, 3.6-, and 5.4fold after Wnt3a application into the maxillary prominence $(P<0.05)$. Thus, these gene expression patterns might correspond to maxillary development (Fig. 6).

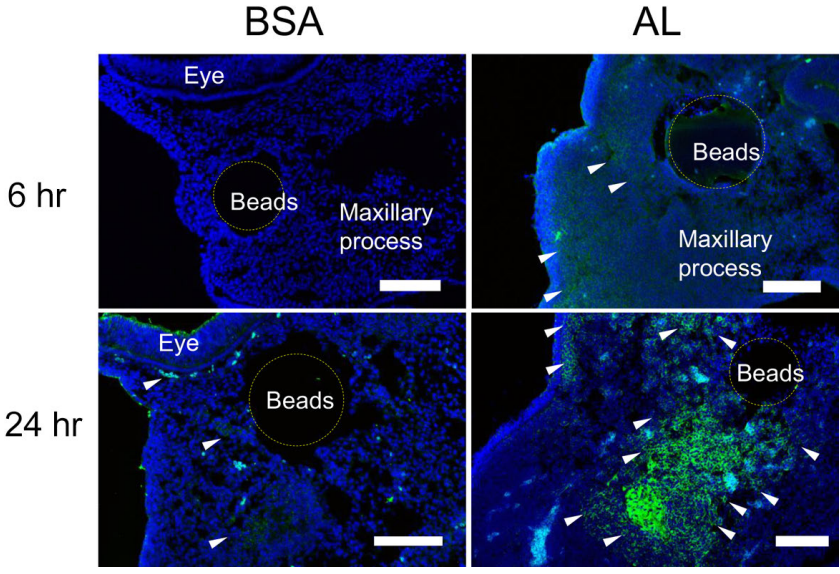

Fig. 5. Expression of $\mathrm{N}$-cadherin in the maxillary prominences of embryos at $6 \mathrm{hr}$ and $24 \mathrm{hr}$ after treatment. $\mathrm{N}$-cadherin immunolabelings (green, white arrowhead) were expressed in the extracellular space around the beads. Nuclei were counterstained with DAPI (blue). The Ncadherin labeling increased following AL-soaked bead implantation in the maxillary prominences at $24 \mathrm{hr}$ after treatment $(\mathrm{n}=3 / 3) . \mathrm{Bar}=100$ $\mu \mathrm{m}$.

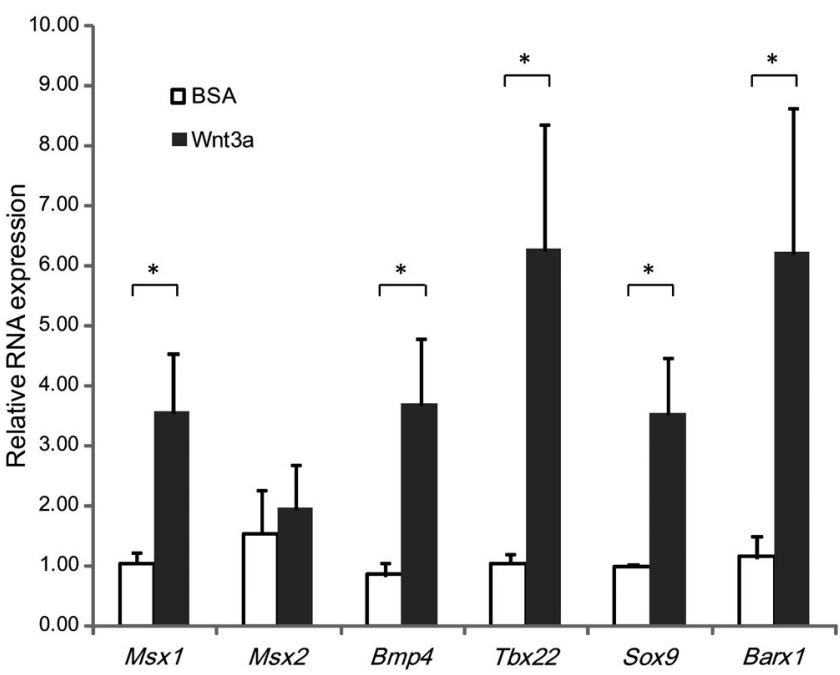

Fig. 6. Real-time RT-PCR analysis of chick maxillary mesenchyme treated with Wnt3a-soaked beads. The chart shows real-time RT-PCR results for different genes from maxillary mesenchyme treated with BSA and Wnt3a. Each bar shows BSA treated sample on the left and experimental situation on the right. Msx 1, Bmp4, Tbx22, Sox9, and Barxl gene expression significantly increased following Wnt3a-soaked bead implantation in the maxillary prominences. Msx 2 mRNA levels showed no significant difference from BSA treatment. Values are the mean \pm S.E. in separate experiments using 9 embryos. ${ }^{*}: P<0.05$, significantly different from the corresponding value for BSA treated group.

\section{Discussion}

Our results provide genetic evidence that $\mathrm{Wnt} / \beta$ catenin signaling regulates maxillary development. We demonstrated that inactivation of the canonical Wnt signaling pathway, via disruption of $\beta$-catenin or of co-receptors of the Wnt signaling pathway, can repress both cell prolif- 
eration at the maxillary prominence and expression of the Bmp4, Sox9, Tbx22, and Barxl genes.

The secreted protein Dkk-1 can negatively regulate Wnt signaling activity $[9,24]$. We found that embryos treated with Dkk-1 showed hypoplasia of the maxilla, including a defect of the maxillary bone, jugal bone and palate bone, which indicated that inhibition of Wnt signaling led to maxillary defects. BrdU-positive cells showed a significant decrease at 24 and $48 \mathrm{hr}$ after treatment, which demonstrating that Dkk-1 inhibited cell proliferation activity. Those observations suggest that the hypoplasia of the maxillary bone results from reduced cell proliferation and differentiation in the maxillary prominences.

We revealed that Bmp4, Sox9, Tbx22, and Barx1 were downstream targets of Wnt signaling. Rescue experiments with AL confirmed those results. BMP proteins, especially BMP4, are known to affect facial shape in avian and mammalian systems [16]. Bmp4 is first expressed in the epithelium of the maxillary and lateral frontonasal prominences in early embryos [1]. BMP4 induces chondrogenesis, the pattern of which is positively regulated by $\operatorname{Sox} 9$ expression $[5,29]$. Sox 9 belongs to the SOX family of transcription factors, and is required for the commitment to cartilage formation, and for differentiation of cartilage cells [19]. We revealed that both Bmp4 and Sox9 expression decreased significantly by blocking the Wnt signaling, which demonstrated that Bmp4 and Sox9 are downstream of the epithelial Wnt signal [5]. As Bmp4 and Sox9 can control osteogenic and chondrogenic cell development, the maxillary defect in the embryo may result from a failure of osteochondrogenesis due to repression of Bmp4 and Sox9. Regarding Tbx22, CLP and ankyloglossia in humans are associated with TBX22 mutations [17]. Ectopic expression of TBX22 in the frontonasal mass results in cleft lip in the chick embryo [12]. TBX proteins function in proliferation, differentiation and the epithelial-mesenchymal transition (EMT) in maxillofacial development $[27,30]$. Thus, the growth defect in chick embryos treated with Dkk-1 probably resulted from down-regulation of TBX function. Barxl is expressed in the mesenchyme of maxillary and mandibular prominences and contributes to pharyngeal osteochondrogenic condensation, which is closely linked to epithelial Bmp 4 [2]. In addition, Barx 1 influences the distribution of ectomesenchymal cells $[32,35]$. In the maxillary prominences, mesenchymal expression of Barxl is complementary to that of Msxl, and Barxl expression increased significantly when Wnt signaling activity increased in response to Wnt $3 a$. As osteochondrogenesis requires the aggregation of progenitor cells in the mesenchyme, the maxillary hypoplasia in our experiments may have resulted from a failure of osteochondrogenic cell condensation due to reduced Barxl expression.

Since Wnt signaling blockage by Dkk-1 downregulated Bmp4 expression, we next examined further transinduction effects resulting from the decreased level of Bmp. Noggin, secreted as a homodimeric glycoprotein, is a BMP antagonist that interferes with the BMPs receptors [5].
When we applied Noggin to the maxillary prominences, $W n t 3 a$ gene expression increased two-fold. This indicated that Noggin can up-regulate Wnt signaling as a feedback loop. Bmp signaling reportedly down-regulates canonical Wnt signaling in osteoblasts $[9,16]$. Thus, it is possible that Wnt signal is negatively controlled by Bmp signals. Indeed, BMPs can act in concert with Wnt antagonists to promote the differentiation of cranial mesodermal cells and thereby promote cranial myogenesis [36]. On the other hand, we found that Noggin increased Bmp4 expression approximately six-fold. This increase in Bmp expression may interact with the decrease in BMP with Noggin application to establish the feedback.

Alsterpaullone is a potent inhibitor of GSK-3 $\beta$ which integrates $\beta$-catenin and stimulates Wnt signaling. When we used AL to enhance Wnt signaling activity, its application significantly increased Msxl expression in the maxillary mesenchyme. Msxl is expressed at high levels in craniofacial skeletal cells during postnatal development. MSX1 protein is essential for cell proliferation and differentiation, and acts as a modeling factor for membranous bone and stimulates trabecular bone metabolism [26]. Our data showed that activation of Wnt signaling by AL upregulated the $M s x l$ gene in the maxillary prominence, but not other genes. Msxl genes play a role in the regulation of $\mathrm{N}$-cadherin-mediated cell adhesion and cell sorting [21]. A previous study has shown that conditional ablation of $\beta$ catenin in the forebrain results in head truncation; this was suggested to be caused by the interaction of $\beta$-catenin with $\mathrm{N}$-cadherin affecting cell adhesion and the maintenance of epithelial architecture of the neuroepithelium $[13,15]$. We found that the expression of N-cadherin protein markedly increased at $24 \mathrm{hr}$ after AL application. N-cadherin is involved in cell agglutination, and chondrogenesis begins because chondrocytes agglutinate [8]. This suggests that Wnt signaling activation by AL lead to N-cadherinmediated chondrocytic agglutination via the Msxl gene.

Since AL did not up-regulate any genes related to craniofacial development except $M s x l$, we treated the embryos with Wnt3a as the ligand of the Wnt signaling pathway. Bmp4, Bmp 7, Tbx22, Sox9, Barxl, as well as Msxl were significantly up-regulated by Wnt3a application whereas were down-regulated by Dkk-1 in the maxillary prominence. These genes were related to skeletal development and craniofacial morphogenesis. Thus, it is likely that Wnt signaling is able to transcript and activate above gene expression and probably specifies some patterning in the maxillary prominences and thereby promotes facial development.

In summary, we find that normal maxillary development is controlled by Bmp4, Tbx22, Sox9, and Barx1 as target genes of the Wnt signaling pathway. Our results also indicate that $\mathrm{Wnt}$ signaling regulates $\mathrm{N}$-cadherin expression via $M s x 1$, resulting in cell aggregation for osteochondrogenesis. 


\section{Conflicts of Interest}

There are no conflicts of interest in connection with this paper.

\section{Acknowledgments}

We thank Ian Smith for manuscript editing. This work was supported by Grant-in-Aid for Scientific Research from JSPS $(24593106,15 \mathrm{~K} 11352,18 \mathrm{~K} 09841)$ to MK.

\section{References}

1. Abzhanov, A., Protas, M., Grant, B. R., Grant, P. R. and Tabin, C. J. (2004) Bmp4 and morphological variation of beaks in Darwin's finches. Science 305; 1462-1465.

2. Barlow, A. J., Bogardi, J. P., Ladher, R. and Francis-West, P. H. (1999) Expression of chick Barx-1 and its differential regulation by FGF-8 and BMP signaling in the maxillary primordia. Dev. Dyn. 214; 291-302.

3. Beaty, T. H., Marazita, M. L. and Leslie, E. J. (2016) Genetic factors influencing risk to orofacial clefts: today's challenges and tomorrow's opportunities. F1000Res. 5; 2800.

4. Broun, M., Gee, L., Reinhardt, B. and Bode, H. R. (2005) Formation of the head organizer in hydra involves the canonical Wnt pathway. Development 132; 2907-2916.

5. Cela, P., Buchtova, M., Vesela, I., Fu, K., Bogardi, J. P., Song, Y., Barlow, A., Buxton, P., Medalova, J., Francis-West, P. and Richman, J. M. (2016) BMP signaling regulates the fate of chondro-osteoprogenitor cells in facial mesenchyme in a stagespecific manner. Dev. Dyn. 245; 947-962.

6. Chenevix-Trench, G., Jones, K., Green, A. C., Duffy, D. L. and Martin, N. G. (1992) Cleft lip with or without cleft palate: associations with transforming growth factor alpha and retinoic acid receptor loci. Am. J. Hum. Genet. 51; 1377-1385.

7. Clevers, H. (2006) Wnt/beta-catenin signaling in development and disease. Cell 127; 469-480.

8. Dady, A. and Duband, J. L. (2017) Cadherin interplay during neural crest segregation from the non-neural ectoderm and neural tube in the early chick embryo. Dev. Dyn. 246; 550-565.

9. Fossat, N., Jones, V., Khoo, P. L., Bogani, D., Hardy, A., Steiner, K., Mukhopadhyay, M., Westphal, H., Nolan, P. M., Arkell, R. and Tam, P. P. (2011) Stringent requirement of a proper level of canonical WNT signalling activity for head formation in mouse embryo. Development 138; 667-676.

10. Hamburger, V. and Hamilton, H. L. (1951) A series of normal stages in the development of the chick embryo. J. Morphol. 88; 49-92.

11. Haraguchi, R., Kitazawa, R., Murashima, A., Yamada, G. and Kitazawa, S. (2017) Developmental contribution of Wnt-signalresponsive cells to mouse reproductive tract formation. Acta Histochem. Cytochem. 50; 127-133.

12. Higashihori, N., Buchtova, M. and Richman, J. M. (2010) The function and regulation of TBX22 in avian frontonasal morphogenesis. Dev. Dyn. 239; 458-473.

13. Howard, S., Deroo, T., Fujita, Y. and Itasaki, N. (2011) A positive role of cadherin in Wnt/beta-catenin signalling during epithelial-mesenchymal transition. PLoS One 6; e23899.

14. Jin, Y. R., Han, X. H., Taketo, M. M. and Yoon, J. K. (2012) Wnt9b-dependent FGF signaling is crucial for outgrowth of the nasal and maxillary processes during upper jaw and lip development. Development 139; 1821-1830.
15. Junghans, D., Hack, I., Frotscher, M., Taylor, V. and Kemler, R. (2005) Beta-catenin-mediated cell-adhesion is vital for embryonic forebrain development. Dev. Dyn. 233; 528-539.

16. Kamiya, N., Ye, L., Kobayashi, T., Mochida, Y., Yamauchi, M., Kronenberg, H. M., Feng, J. Q. and Mishina, Y. (2008) BMP signaling negatively regulates bone mass through sclerostin by inhibiting the canonical Wnt pathway. Development 135; 38013811.

17. Kantaputra, P. N., Paramee, M., Kaewkhampa, A., Hoshino, A., Lees, M., McEntagart, M., Masrour, N., Moore, G. E., Pauws, E. and Stanier, P. (2011) Cleft lip with cleft palate, ankyloglossia, and hypodontia are associated with TBX22 mutations. J. Dent. Res. 90; 450-455.

18. Kawakami, M., Okuda, H., Tatsumi, K., Kirita, T. and Wanaka, A. (2014) Inhibition of Wnt/beta-catenin pathway by Dickkopf-1 affects midfacial morphogenesis in chick embryo. J. Biosci. Bioeng. 117; 664-669.

19. Kishi, S., Abe, H., Akiyama, H., Tominaga, T., Murakami, T., Mima, A., Nagai, K., Kishi, F., Matsuura, M., Matsubara, T., Iehara, N., Ueda, O., Fukushima, N., Jishage, K. and Doi, T. (2011) SOX9 protein induces a chondrogenic phenotype of mesangial cells and contributes to advanced diabetic nephropathy. J. Biol. Chem. 286; 32162-32169.

20. Lan, Y., Ryan, R. C., Zhang, Z., Bullard, S. A., Bush, J. O., Maltby, K. M., Lidral, A. C. and Jiang, R. (2006) Expression of Wnt9b and activation of canonical Wnt signaling during midfacial morphogenesis in mice. Dev. Dyn. 235; 1448-1454.

21. Lincecum, J. M., Fannon, A., Song, K., Wang, Y. and Sassoon, D. A. (1998) Msh homeobox genes regulate cadherin-mediated cell adhesion and cell-cell sorting. J. Cell. Biochem. 70; 22-28.

22. Liu, F. and Millar, S. E. (2010) Wnt/beta-catenin signaling in oral tissue development and disease. J. Dent. Res. 89; 318-330.

23. Liu, W., Sun, X., Braut, A., Mishina, Y., Behringer, R. R., Mina, M. and Martin, J. F. (2005) Distinct functions for Bmp signaling in lip and palate fusion in mice. Development $132 ; 1453-1461$.

24. MacDonald, B. T., Tamai, K. and He, X. (2009) Wnt/betacatenin signaling: components, mechanisms, and diseases. Dev. Cell 17; 9-26.

25. Mossey, P. A., Little, J., Munger, R. G., Dixon, M. J. and Shaw, W. C. (2009) Cleft lip and palate. Lancet 374; 1773-1785.

26. Nassif, A., Senussi, I., Meary, F., Loiodice, S., Hotton, D., Robert, B., Bensidhoum, M., Berdal, A. and Babajko, S. (2014) Msx1 role in craniofacial bone morphogenesis. Bone 66; 96-104.

27. Papaioannou, V. E. (2014) The T-box gene family: emerging roles in development, stem cells and cancer. Development 141; 3819-3833.

28. Reid, B. S., Yang, H., Melvin, V. S., Taketo, M. M. and Williams, T. (2011) Ectodermal Wnt/beta-catenin signaling shapes the mouse face. Dev. Biol. 349; 261-269.

29. Semba, I., Nonaka, K., Takahashi, I., Takahashi, K., Dashner, R., Shum, L., Nuckolls, G. H. and Slavkin, H. C. (2000) Positionally-dependent chondrogenesis induced by BMP4 is coregulated by Sox 9 and Msx2. Dev. Dyn. 217; 401-414.

30. Shimoda, M., Sugiura, T., Imajyo, I., Ishii, K., Chigita, S., Seki, K., Kobayashi, Y. and Shirasuna, K. (2012) The T-box transcription factor Brachyury regulates epithelial-mesenchymal transition in association with cancer stem-like cells in adenoid cystic carcinomafactor Brachyury regulates epithelialmesenchymal transition in association with cancer stem-like cells in adenoid cystic carcinoma cells. BMC Cancer 12; 377.

31. Song, L., Li, Y., Wang, K., Wang, Y. Z., Molotkov, A., Gao, L., Zhao, T., Yamagami, T., Wang, Y., Gan, Q., Pleasure, D. E. and Zhou, C. J. (2009) Lrp6-mediated canonical Wnt signaling is required for lip formation and fusion. Development 136; 31613171 . 
32. Sperber, S. M. and Dawid, I. B. (2008) barx1 is necessary for ectomesenchyme proliferation and osteochondroprogenitor condensation in the zebrafish pharyngeal arches. Dev. Biol. 321; $101-110$.

33. Szabo-Rogers, H. L., Geetha-Loganathan, P., Nimmagadda, S., $\mathrm{Fu}, \mathrm{K}$. K. and Richman, J. M. (2008) FGF signals from the nasal pit are necessary for normal facial morphogenesis. Dev. Biol. 318; 289-302.

34. Szabo-Rogers, H. L., Smithers, L. E., Yakob, W. and Liu, K. J. (2010) New directions in craniofacial morphogenesis. Dev. Biol. 341; 84-94.

35. Tak, H. J., Park, T. J., Piao, Z. and Lee, S. H. (2017) Separate development of the maxilla and mandible is controlled by regional signaling of the maxillomandibular junction during avian development. Dev. Dyn. 246; 28-40.
36. Tzahor, E., Kempf, H., Mootoosamy, R. C., Poon, A. C., Abzhanov, A., Tabin, C. J., Dietrich, S. and Lassar, A. B. (2003) Antagonists of Wnt and BMP signaling promote the formation of vertebrate head muscle. Genes Dev. 17; 3087-3099.

37. Wang, Y., Song, L. and Zhou, C. J. (2011) The canonical Wnt/ beta-catenin signaling pathway regulates Fgf signaling for early facial development. Dev. Biol. 349; 250-260.

38. Zhu, X. J., Liu, Y., Yuan, X., Wang, M., Zhao, W., Yang, X., Zhang, X., Hsu, W., Qiu, M., Zhang, Z. and Zhang, Z. (2016) Ectodermal Wnt controls nasal pit morphogenesis through modulation of the BMP/FGF/JNK signaling axis. Dev. Dyn. 245; 414-426.

This is an open access article distributed under the Creative Commons Attribution License, which permits unrestricted use, distribution, and reproduction in any medium, provided the original work is properly cited. 